\title{
The Structural Phase Transitions in 6CB-Based Ferronematics
}

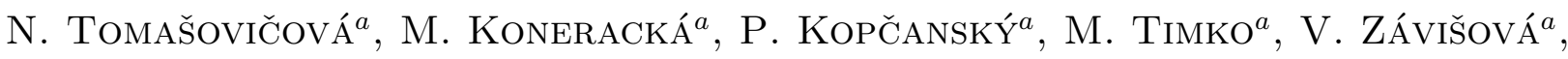 \\ L. TOMČO ${ }^{b}$ AND J. JADŻYN ${ }^{c}$ \\ ${ }^{a}$ Institute of Experimental Physics, Slovak Academy of Sciences \\ Watsonová 47, 04001 Košice, Slovakia \\ ${ }^{b}$ Faculty of Aeronautics, Technical University \\ Rampová 7, 04121 Košice, Slovakia \\ ${ }^{c}$ Institute of Molecular Physics, Polish Academy of Sciences \\ M. Smoluchowskiego 17, 60-179 Poznań, Poland
}

\begin{abstract}
The structural transitions in ferronematics based on the thermotropic nematic liquid crystal 4-cyano-4'-hexylbiphenyl were studied. The ferronematic samples were prepared by doping with magnetic suspension consisting of $\mathrm{Fe}_{3} \mathrm{O}_{4}$ particles (10 nm in diameter) coated with oleic acid as a surfactant with different volume concentrations of magnetic particles (from $10^{-5}$ to $10^{-3}$ ). Freedericksz transitions were studied in combined electric and magnetic fields. The experimental results indicated soft anchoring with perpendicular initial orientation between the magnetic moment of the magnetic particles and director.
\end{abstract}

PACS numbers: 75.50.Mm, 61.30.Gd, 61.30.Hn

\section{Introduction}

The liquid crystals can be oriented under magnetic or electric fields due to their anisotropic properties. However, because of small value of the anisotropy of diamagnetic susceptibility $\left(\chi_{\mathrm{a}} \approx 10^{-7}\right)$, a magnetic field necessary to align liquid crystals have to reach rather large values $(B \approx 1 \mathrm{~T})$. In effort to enhance the magnetic susceptibility of liquid crystals, the idea of doping them with fine magnetic particles was theoretically introduced by Brochard and de Gennes [1]. Recently, the complex systems containing nanoparticles gained a great scientific interest. Especially, the ferronematics exhibit complex features. These systems are thermotropic liquid crystals, in which magnetic particles are suspended colloidally. So in presence of a magnetic field the liquid crystal molecules are easily oriented when they are doped with magnetic particles. Number of features has been attributed to a coupling between the magnetic particles and the liquid crystal director $\boldsymbol{n}$ which denotes the preferential direction of the nematic molecules. Brochard and de Gennes [1] predicted that a rigid anchoring $\boldsymbol{m} \| \boldsymbol{n}$, where the unit vector $\boldsymbol{m}$ denotes orientation of the magnetic moment of the magnetic particles, would result in the ferromagnetic behavior of the nematic matrix. Based on the experiments, which excluded the presence of parallel orientation of $\boldsymbol{m}$ and $\boldsymbol{n}$ in thermotropic ferronematics, the Burylov and Raikher theory was constructed $[2-4]$. This theory considers the finite value of the surface density of anchoring energy $W$ at the nematic-magnetic particle boundary. The finite value of $W$, as well as the parameter $\omega$ that is defined as a ratio of anchoring energy to elastic energy of liquid crystal $(\omega=W d / K$, where $d$ is size of the magnetic particles and $K$ is orientational-elastic Frank modulus), characterize the type of anchoring of nematic molecules on magnetic particles surfaces. The parameter $\omega \gg 1$ characterize the rigid anchoring. The soft anchoring, characterized by parameter $\omega \leq 1$, unlike the rigid, permits both types of boundary conditions ( $\boldsymbol{m} \| \boldsymbol{n}$ and $\boldsymbol{m} \perp \boldsymbol{n}$ ), thus the Burylov and Raikher theory could be applied for thermotropic ferronematics. The main purpose of our study is devoted to the changes in the behavior of the liquid crystal matrix in external magnetic field by the addition of different volume concentration of magnetic particles.

\section{Experimental}

The synthesis of the spherical magnetic nanoparticles was based on co-precipitation of $\mathrm{Fe}^{2+}$ and $\mathrm{Fe}^{3+}$ salts by $\mathrm{NH}_{4} \mathrm{OH}$ at $60^{\circ} \mathrm{C}$. In order to prepare stable suspension of magnetite particles they were coated by oleic acid and dispersed in well known thermotropic liquid crystal 4- $n$-hexyl-4'-cyanobiphenyl (6CB). 6CB, as well as other members of the $n \mathrm{CB}$ homologous series, is important from the point of view of applications due to the pos- 
session of a strong dipole moment, good chemical stability and a convenient temperature range of the nematic phase [5]. The doping was simply done by adding the suspension of magnetic particles, under continuous stirring, to the liquid crystal in isotropic phase. The small volume concentrations of the magnetic particles $(\phi \sim$ $10^{-5}-10^{-3}$ ) and the surfactant in the prepared ferronematic samples should avoid the interparticle dipoledipole interactions. The homogeneity and stability of the samples were verified by optical microscopy and by dielectric measurements indirectly [6]. The structural transitions in ferronematic samples were indicated by capacitance measurements in a capacitor with ITO-coated glass electrodes (LINCAM co.). The capacitor with the electrode area approximately $1 \mathrm{~cm} \times 1 \mathrm{~cm}$ was connected to a regulated thermostat system, the temperature was stabilized with the accuracy of $0.05^{\circ} \mathrm{C}$. The distance between the electrodes (sample thickness) was $D=5 \mu \mathrm{m}$. The capacitance was measured at the frequency $1 \mathrm{kHz}$ by the high precision capacitance bridge Andeen Hagerling. The stability of the samples in the strong magnetic fields was verified by repeating the capacitance measurements after 5 months on the same samples, with reproducible results.

\section{Results and discussion}

The surface of the magnetic particles is able to orient the adjacent liquid crystal molecules. The strength of that anchoring is characterized by the parameter $\omega$. The observations of the structural transitions in ferronematics in external fields can be used for determination of the type of anchoring of nematic molecules on magnetic particle surfaces as well as the surface density of the anchoring energy $W$ at the nematic-magnetic particle boundary. During measurements the bias electric field was applied perpendicular to the capacitor electrodes and the external magnetic field was applied perpendicular to the bias electric field (Fig. 1). The dependence of the measured capacitance on the external magnetic field reflects the re-orientation of the nematic molecules in the strong magnetic field. Figure 2 shows the dependence of the capacitance of pure 6CB liquid crystal and 6CB liquid crystal doped with spherical magnetic particles $\left(\phi_{3}=10^{-3}\right)$ on the external magnetic field at different bias voltage. From this figure it is seen that the critical magnetic field, i.e. the magnetic field that turns the molecules of liquid crystal toward its direction, is shifted to higher values with increasing bias voltage. The similar dependences were observed for all samples. By means of the Burylov and Raikher expression for the free energy of ferronematic [4] the formula for the critical magnetic field was estimated as follows:

$$
B_{\mathrm{CFN}}^{2}-B_{\mathrm{C}}^{2}=\frac{\epsilon_{0} \epsilon_{\mathrm{a}} \mu_{0} U_{\mathrm{B}}^{2}}{D^{2}}+\frac{2 \mu_{0} W \phi}{\chi_{\mathrm{a}} d},
$$

where $B_{\mathrm{C}}$ and $B_{\mathrm{CFN}}$ are the critical fields of magnetic Fredericksz transition of pure liquid crystal and ferronematic, respectively, $U_{\mathrm{B}}$ is the applied electric field, $\epsilon_{0}$ is

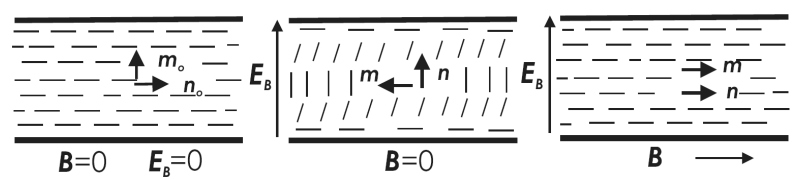

Fig. 1. Cross-section of the cell in the initial state, after application of the electric field $E_{\mathrm{B}}>E_{\mathrm{C}}$ and after application of the external magnetic field $B>B_{\mathrm{C}}$.

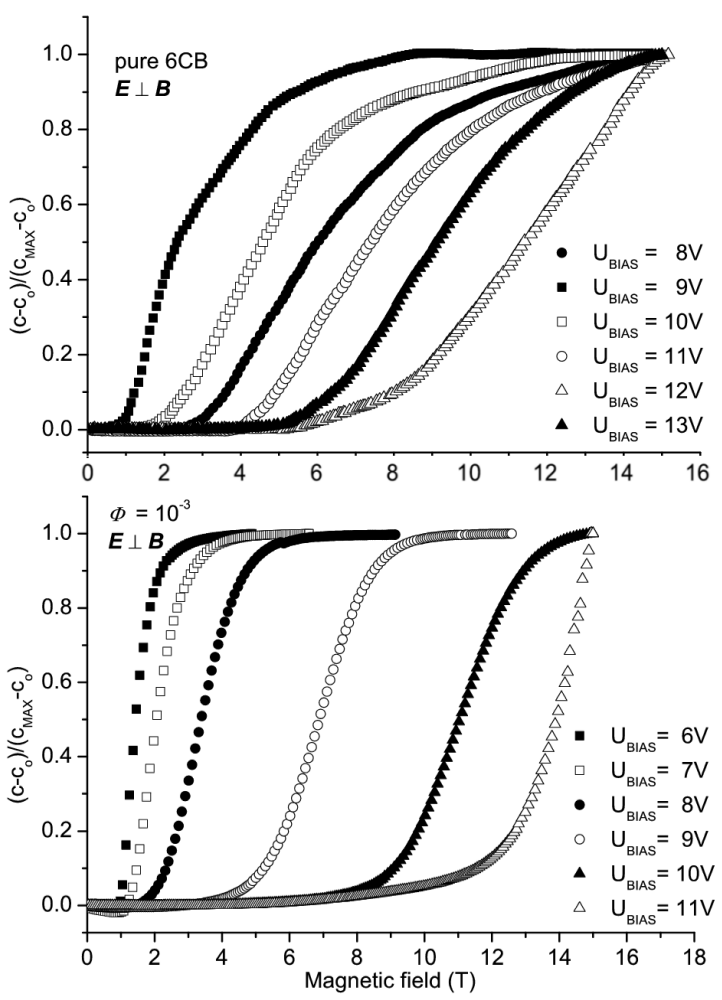

Fig. 2. Capacitance dependence of pure $6 \mathrm{CB}$ and $6 \mathrm{CB}$ doped with magnetic particles $\left(\phi_{3}=10^{-3}\right)$ on external magnetic field measured at different bias voltage.

the permittivity of vacuum, $\epsilon_{\mathrm{a}}$ is the anisotropy of dielectric permittivity, $D$ is the thickness of the sample, $d$ is the mean diameter of spherical particles, $\phi$ is the volume concentration of magnetic particles in liquid crystal, $\mu_{0}$ is the permeability of vacuum and $\chi_{\mathrm{a}}$ is anisotropy of diamagnetic susceptibility of liquid crystal. The calculated value of surface density of anchoring energy $W$ at the nematic-magnetic particle boundary at the temperature $T=25^{\circ} \mathrm{C}$ is order of $10^{-1} \mathrm{~N} \mathrm{~m}^{-1}$ and the obtained values of $W$ were used for calculation of the parameter $\omega$ (for pure $6 \mathrm{CB}$ is $K_{1}=431.5 \mathrm{pN}$ ). The obtained value $\omega$ is of order of $10^{-1} \mathrm{~N} \mathrm{~m}^{-1}$ that characterizes soft anchoring of nematic molecules on the surfaces of the magnetic particles that permits both types of boundary conditions ( $\boldsymbol{m} \| \boldsymbol{n}$ and $\boldsymbol{m} \perp \boldsymbol{n}$ ). The applied bias electric voltage $U_{\mathrm{B}}$ induces a splay Freedericksz transition, rotating the nematic molecules normal to the electrodes. The increasing external magnetic field $\boldsymbol{B}$ reduces this deformation by 


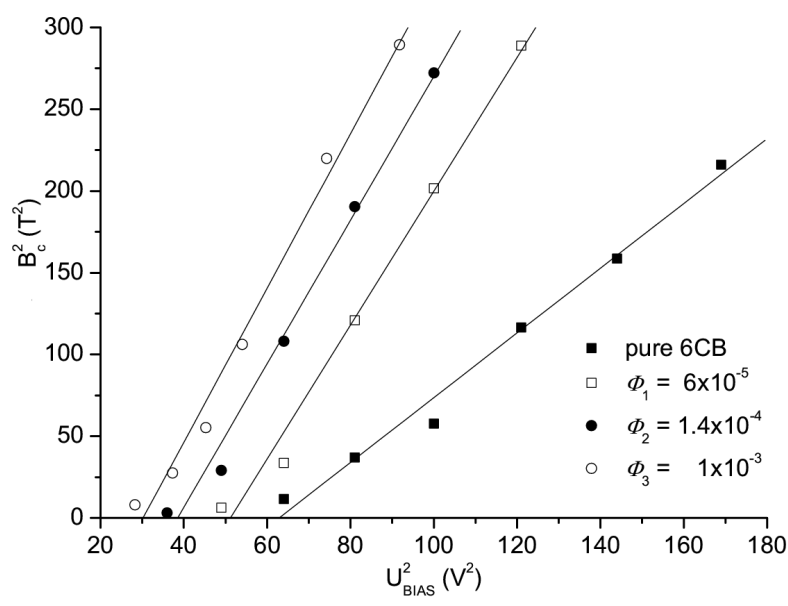

Fig. 3. Dependence of critical magnetic field on the bias voltage for pure $6 \mathrm{CB}$ and $6 \mathrm{CB}$ doped with magnetic particles for different volume concentrations.

turning the director back toward the magnetic field. At the threshold magnetic field $B_{\mathrm{c}}$ the initial planar texture is restored, i.e. the electric Freedericksz transition is fully inhibited. In this case the addition of fine magnetic particles increases $B_{\mathrm{c}}$. Figure 3 shows dependence of square of critical magnetic field on the square of applied bias voltage for pure $6 \mathrm{CB}$ and $6 \mathrm{CB}$ doped with spherical magnetic particles for different volume concentrations and the best linear fits according to the theoretical model. From this figure is seen that critical magnetic field is increased with increasing volume concentration of magnetic parti- cles which means that the initial boundary condition in 6CB-based ferronematics is perpendicular, because the magnetic field has to overcome anchoring energy between molecules of liquid crystal and magnetic particles.

\section{Acknowledgments}

This work was supported by the Slovak Academy of Sciences (grant No. 0077), Slovak Research and Development Agency under the contract No. APVV-SK-MAD026-06 and APVV-0509-07, the Grenoble High Magnetic Field Laboratory, with support of EC Program RITACT-2003-505474.

\section{References}

[1] F. Brochard, P.G. de Gennes, J. Phys. (Paris) 31, 691 (1970).

[2] S.V. Burylov, Y.L. Raikher, J. Phys. Lett. A 149, 279 (1990).

[3] S.V. Burylov, Y.L. Raikher, J. Magn. Magn. Mater. 122, 62 (1993).

[4] S.V. Burylov, Y.L. Raikher, Mol. Cryst. Liq. Cryst. 255, 107 (1995).

[5] J. Czub, S. Urban, A. Wurflinger, Liq. Cryst. 33, 85 (2006).

[6] P. Kopčanský, M. Koneracká, M. Timko, I. Potočová, L. Tomčo, N. Tomašovičová, V. Závišová, J. Jadzyn, J. Magn. Magn. Mater. 300, 75 (2006). 